\title{
Clinical trial recruiters' experiences working with trial eligibility criteria: results of an exploratory, cross-sectional, online survey in the UK
}

William J. Cragg ${ }^{*} \mathbb{D}$, Kathryn McMahon, Jamie B. Oughton®D, Rachel Sigsworth, Christopher Taylor and Vicky Napp(D)

\begin{abstract}
Background: Eligibility criteria are a fundamental element of clinical trial design, defining who can and who should not participate in a trial. Problems with the design or application of criteria are known to occur and pose risks to participants' safety and trial integrity, sometimes also negatively impacting on trial recruitment and generalisability. We conducted a short, exploratory survey to gather evidence on UK recruiters' experiences interpreting and applying eligibility criteria and their views on how criteria are communicated and developed.

Methods: Our survey included topics informed by a wider programme of work at the Clinical Trials Research Unit, University of Leeds, on assuring eligibility criteria quality. Respondents were asked to answer based on all their trial experience, not only on experiences with our trials. The survey was disseminated to recruiters collaborating on trials run at our trials unit, and via other mailing lists and social media. The quantitative responses were descriptively analysed, with inductive analysis of free-text responses to identify themes.

Results: A total of 823 eligible respondents participated. In total, $79 \%$ of respondents reported finding problems with eligibility criteria in some trials, and $9 \%$ in most trials. The main themes in the types of problems experienced were criteria clarity (67\% of comments), feasibility (34\%), and suitability (14\%). In total, $27 \%$ of those reporting some level of problem said these problems had led to patients being incorrectly included in trials; $40 \%$ said they had led to incorrect exclusions. Most respondents (56\%) reported accessing eligibility criteria mainly in the trial protocol. Most respondents (74\%) supported the idea of recruiter review of eligibility criteria earlier in the protocol development process.

Conclusions: Our survey corroborates other evidence about the existence of suboptimal trial eligibility criteria. Problems with clarity were the most often reported, but the number of comments on feasibility and suitability suggest some recruiters feel eligibility criteria and associated assessments can hinder recruitment to trials. Our proposal for more recruiter involvement in protocol development has strong support and some potential benefits, but questions remain about how best to implement this. We invite other trialists to consider our other suggestions for how to assure quality in trial eligibility criteria.
\end{abstract}

\footnotetext{
* Correspondence: w.cragg@leeds.ac.uk

Clinical Trials Research Unit, Leeds Institute of Clinical Trials Research,

University of Leeds, Leeds LS2 9JT, UK
}

(c) The Author(s). 2021, corrected publication 2021. Open Access This article is licensed under a Creative Commons Attribution 4.0 International License, which permits use, sharing, adaptation, distribution and reproduction in any medium or format, as long as you give appropriate credit to the original author(s) and the source, provide a link to the Creative Commons licence, and indicate if changes were made. The images or other third party material in this article are included in the article's Creative Commons licence, unless indicated otherwise in a credit line to the material. If material is not included in the article's Creative Commons licence and your intended use is not permitted by statutory regulation or exceeds the permitted use, you will need to obtain permission directly from the copyright holder. To view a copy of this licence, visit http://creativecommons.org/ licenses/by/4.0/. The Creative Commons Public Domain Dedication waiver (http://creativecommons.org/publicdomain/zero/1. 0/) applies to the data made available in this article, unless otherwise stated in a credit line to the data. 
Keywords: Eligibility criteria, Inclusion criteria, Exclusion criteria, Recruitment, Protocol development, Generalisability, Quality assurance

\section{Administrative information}

\begin{tabular}{|c|c|c|}
\hline Title: & \multicolumn{2}{|c|}{$\begin{array}{l}\text { Clinical trial recruiters' experiences working with trial eligibility } \\
\text { criteria: results of an exploratory, cross-sectional, online survey } \\
\text { in the UK }\end{array}$} \\
\hline Running Head: & \multicolumn{2}{|c|}{ Recruiters' experiences with eligibility criteria } \\
\hline Version + Date: & \multicolumn{2}{|c|}{ Version 2.0 11-Oct-2021 } \\
\hline Word count: & \multicolumn{2}{|l|}{4581} \\
\hline Tables/Figures: & \multicolumn{2}{|l|}{2 tables, 4 figures } \\
\hline Supplement: & \multicolumn{2}{|c|}{$\begin{array}{l}\text { Eligibility criteria survey text } \\
\text { Eligibility criteria survey invitation text } \\
\text { CROSS checklist }\end{array}$} \\
\hline \multirow[t]{7}{*}{ Authors: } & Name & Affiliation \\
\hline & $\begin{array}{l}\text { William J Cragg } \\
\text { w.cragg@leeds. } \\
\text { ac.uk } \\
\text { ORCID: 0000- } \\
\text { 0002-1274-8521 }\end{array}$ & $\begin{array}{l}\text { Clinical Trials Research Unit, Leeds Institute } \\
\text { of Clinical Trials Research, University of } \\
\text { Leeds, UK }\end{array}$ \\
\hline & $\begin{array}{l}\text { Kathryn } \\
\text { McMahon }\end{array}$ & $\begin{array}{l}\text { Clinical Trials Research Unit, Leeds Institute } \\
\text { of Clinical Trials Research, University of } \\
\text { Leeds, UK }\end{array}$ \\
\hline & $\begin{array}{l}\text { Jamie B } \\
\text { Oughton } \\
\text { ORCID: 0000- } \\
\text { 0002-2047- } \\
804 X\end{array}$ & $\begin{array}{l}\text { Clinical Trials Research Unit, Leeds Institute } \\
\text { of Clinical Trials Research, University of } \\
\text { Leeds, UK }\end{array}$ \\
\hline & $\begin{array}{l}\text { Rachel } \\
\text { Sigsworth }\end{array}$ & $\begin{array}{l}\text { Clinical Trials Research Unit, Leeds Institute } \\
\text { of Clinical Trials Research, University of } \\
\text { Leeds, UK }\end{array}$ \\
\hline & $\begin{array}{l}\text { Christopher } \\
\text { Taylor }\end{array}$ & $\begin{array}{l}\text { Clinical Trials Research Unit, Leeds Institute } \\
\text { of Clinical Trials Research, University of } \\
\text { Leeds, UK }\end{array}$ \\
\hline & $\begin{array}{l}\text { Vicky Napp } \\
\text { ORCID: } 0000- \\
\text { 0001-6726-2222 }\end{array}$ & $\begin{array}{l}\text { Clinical Trials Research Unit, Leeds Institute } \\
\text { of Clinical Trials Research, University of } \\
\text { Leeds, UK }\end{array}$ \\
\hline Correspondence: & \multicolumn{2}{|c|}{$\begin{array}{l}\text { William Cragg } \\
\text { w.cragg@leeds.ac.uk } \\
\text { Tel: } 01133438398 \\
\text { Clinical Trials Research Unit } \\
\text { Leeds Institute of Clinical Trials Research } \\
\text { University of Leeds } \\
\text { Leeds } \\
\text { LS2 9JT, } \\
\text { United Kingdom }\end{array}$} \\
\hline $\begin{array}{l}\text { Research } \\
\text { support: }\end{array}$ & \multicolumn{2}{|c|}{$\begin{array}{l}\text { This work was supported by a Cancer Research UK Core CTU } \\
\text { infrastructure grant (reference A24929). }\end{array}$} \\
\hline $\begin{array}{l}\text { Summary of } \\
\text { figures and } \\
\text { tables }\end{array}$ & \multicolumn{2}{|c|}{$\begin{array}{l}\text { Figure 1: characteristics of eligible survey respondents }(n= \\
823 \text { ) } \\
\text { Figure 2: reported incidence of encountering problems with } \\
\text { trial eligibility criteria. } \\
\text { Figure 3: respondents' reported primary method for accessing } \\
\text { trial eligibility criteria } \\
\text { Figure 4: respondents' views on the possibility of earlier trial } \\
\text { protocol review } \\
\text { Table 1: Top } 10 \text { most frequent responses about types of } \\
\text { problems experienced when using eligibility criteria } \\
\text { Table 2: Top } 10 \text { most frequent additional comments about } \\
\text { how eligibility criteria are developed or used }\end{array}$} \\
\hline
\end{tabular}

\section{Background}

Eligibility criteria, also known as inclusion and exclusion criteria, are a fundamental element of clinical trial design [1]. They define who can take part in the research, and who should not, thereby communicating who is expected to benefit from the trial intervention and who should not take part in the trial due to an unfavourable individual risk-benefit ratio [2]. They also indicate the extent to which a trial's results may be generalisable outside the trial population and therefore whether the trial has an explanatory or pragmatic objective [3].

We suggest that optimal eligibility criteria are both (a) well-selected, in that they are collectively necessary and sufficient to help the trial achieve its objectives and protect patients in and outside the trial and (b) well-written, in that the intended meaning of each well-selected criterion is correctly and unambiguously conveyed to everyone who needs to understand it and, as far as possible, uniformly interpreted.

Suboptimal selection or writing of criteria poses risks to research quality and may reduce the ability of eligibility criteria to perform the roles outlined above. Misinterpretation of criteria leading to erroneous enrolment of participants who are not eligible can put those people's safety and wellbeing at risk [4], or can undermine a trial's integrity if many instances occur [5, 6]. Statistical challenges can arise both from the presence of ineligible patients in a trial cohort $[7,8]$, and from protocol amendments required to modify eligibility criteria partway through a trial [9]. Criteria that are designed, written or interpreted in ways that exclude a large proportion of people with the condition of interest may reduce the generalisability of a trial's results [10] and may be unethical in denying people access to research participation and its associated benefits [11, 12]. Overly exclusive criteria may even mean trials exclude those most affected by a particular condition, as others have observed in context of the COVID-19 pandemic [13].

In multi-centre trials, there is a risk that different sites will interpret the eligibility criteria differently. Although this can be accounted for in the trial design through the common practice of using centre as a stratification factor in the randomisation [14], different interpretations of eligibility criteria are still undesirable. A trial's eligibility criteria should be selected to achieve a balance between restrictiveness, to boost statistical power and protect patients, and permissiveness, to increase generalisability 


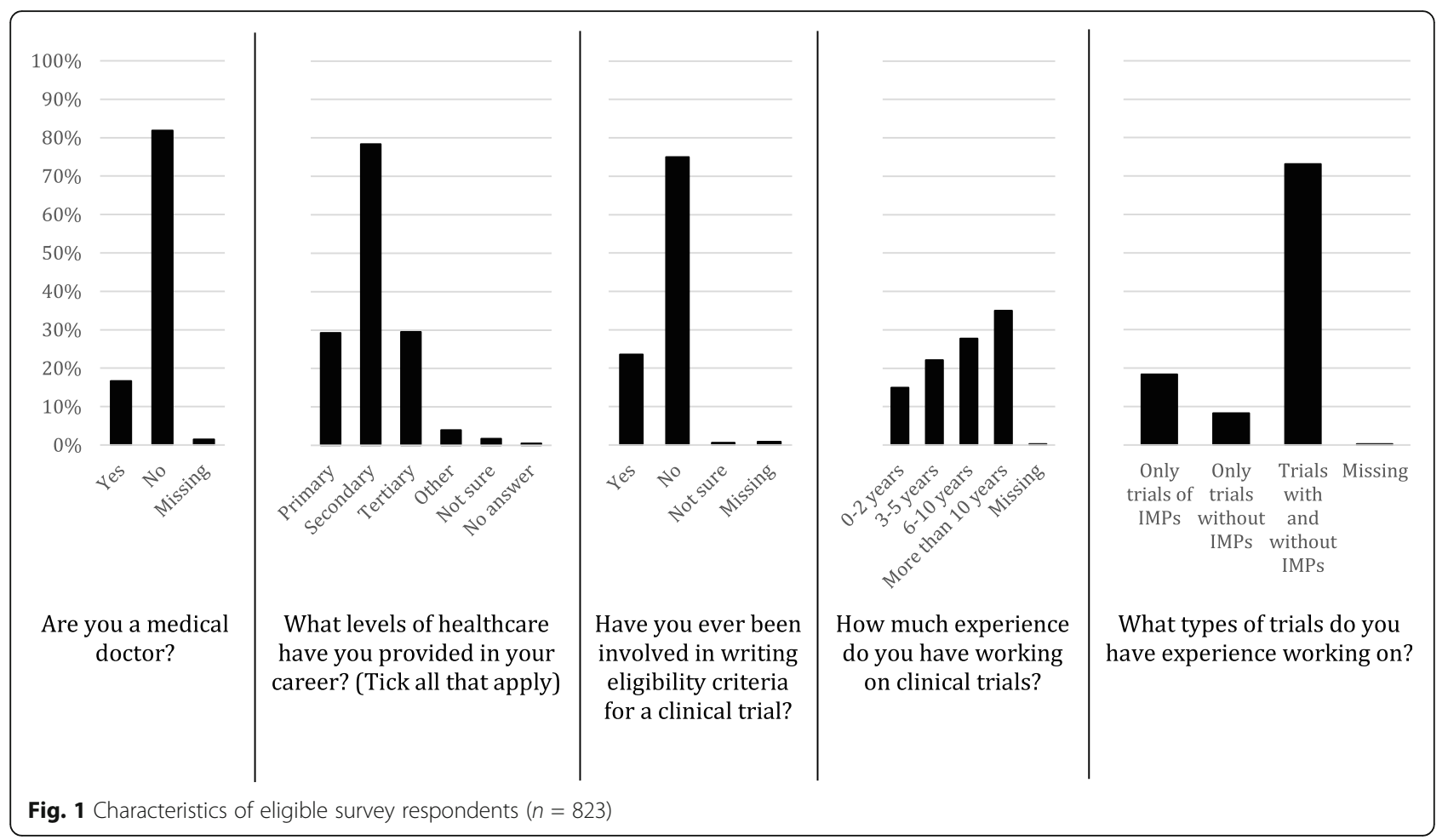

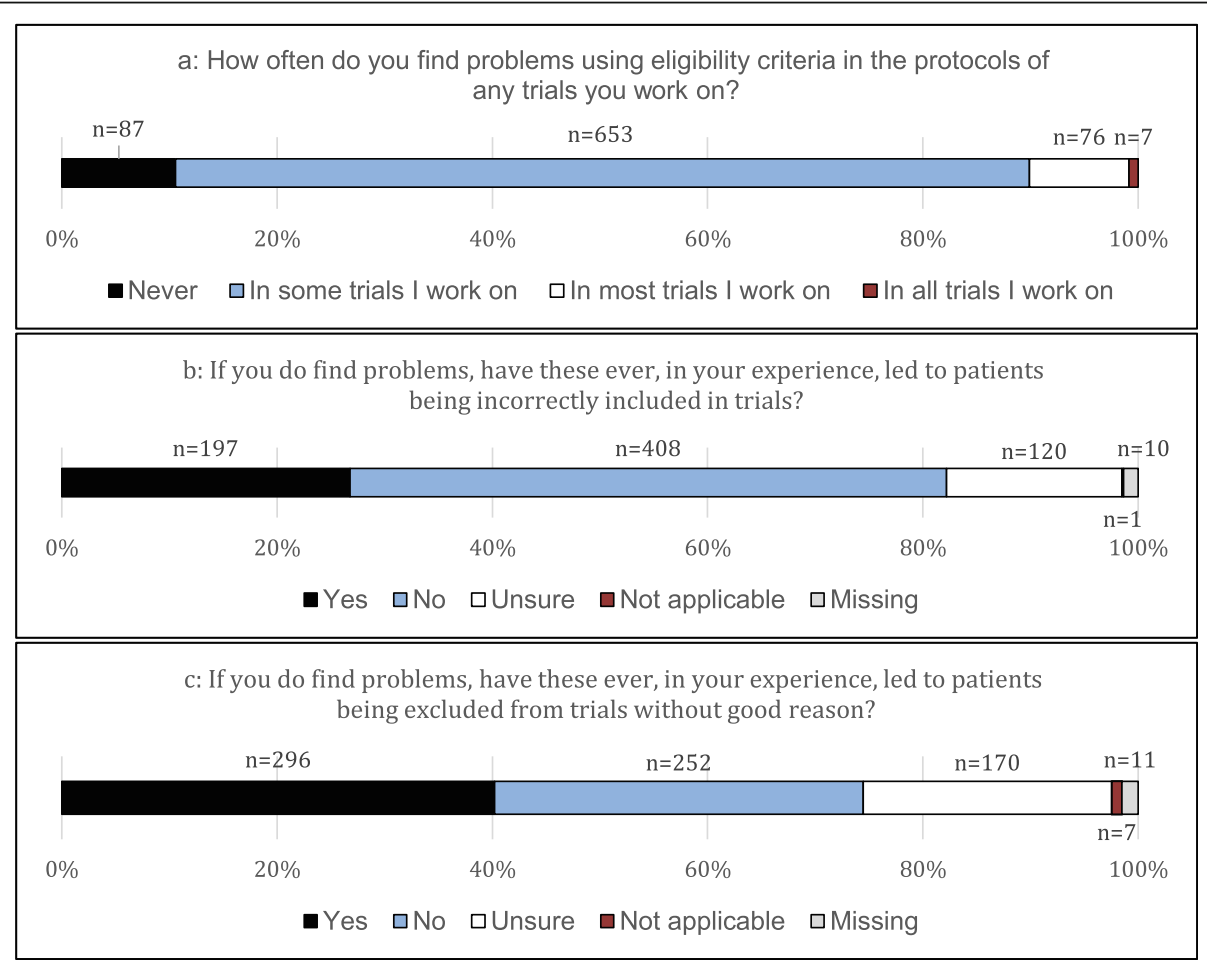

Fig. 2 Reported incidence of encountering problems with trial eligibility criteria. a Incidence in general. b Frequency of incorrect inclusions. c Frequency of incorrect exclusions 


\begin{tabular}{|l}
\hline $\begin{array}{l}\text { How do you most often access eligibility criteria when you are screening a } \\
\text { patient for a trial? }\end{array}$ \\
$\square$ Refer to the protocol \\
\\
$\square$ Refer to eligibility checklist or Case Report Form (CRF) provided by Sponsor or \\
Clinical Trials Unit \\
$\square$ Refer to locally-produced forms (e.g. 'crib sheets') based on the protocol \\
$\square$ Other \\
$\square$ Missing
\end{tabular}

and facilitate recruitment [2, 15-17]. Site-level differences in interpretations represent deviations from this balance. If some sites interpret criteria too strictly, recruitment and generalisability may be negatively affected. If some interpret too loosely, eligibility-related protocol violations may occur, with the resulting statistical challenges and potential impact on patients' safety mentioned above.

Clear communication of eligibility criteria is important in trial reporting, to aid reproducibility and the correct interpretation of trial results [18-20]. Problems in how criteria are written can also be an efficiency issue. Suboptimal criteria may take time to remedy through protocol amendments [21, 22] and may use up more staff time (both at trials units and at recruiting sites) in dealing with queries and uncertainty.

Problems with applying criteria are known to occur in practice $[4,5,23-28]$. Clearly, it can be difficult to foresee all potential problems, but we should nonetheless make all reasonable efforts to build in quality from the outset [29]. There is also an expectation from regulators that trial sponsors take all necessary action to prevent, and monitor the occurrence of, problems arising from eligibility criteria [30].
Eligibility criteria are typically chosen and written by members of a trial management group (led by the Chief Investigator) and included in a trial protocol. In multicentre trials, the protocol is then shared with trial sites where recruiters-after all the required trial approvals are in place-use the eligibility criteria to assess patients for potential suitability for the trial. There is limited information about recruiters' experiences interpreting and applying eligibility criteria, or about their views on how eligibility criteria are communicated and developed. We carried out a short, exploratory survey to gather new, primary evidence.

\section{Methods}

We designed a short, cross-sectional, online survey to gather information on UK clinical trial recruiters' experiences using eligibility criteria, as well as their views on how we might best communicate and collaborate with them. Given the risk of reduced response rate for longer surveys, we deliberately chose to include only a few key questions and to prioritise number of responses rather than information depth. The full survey text is available with the Supplementary Information to this article. We used Jisc Online Surveys [31] to host the survey.

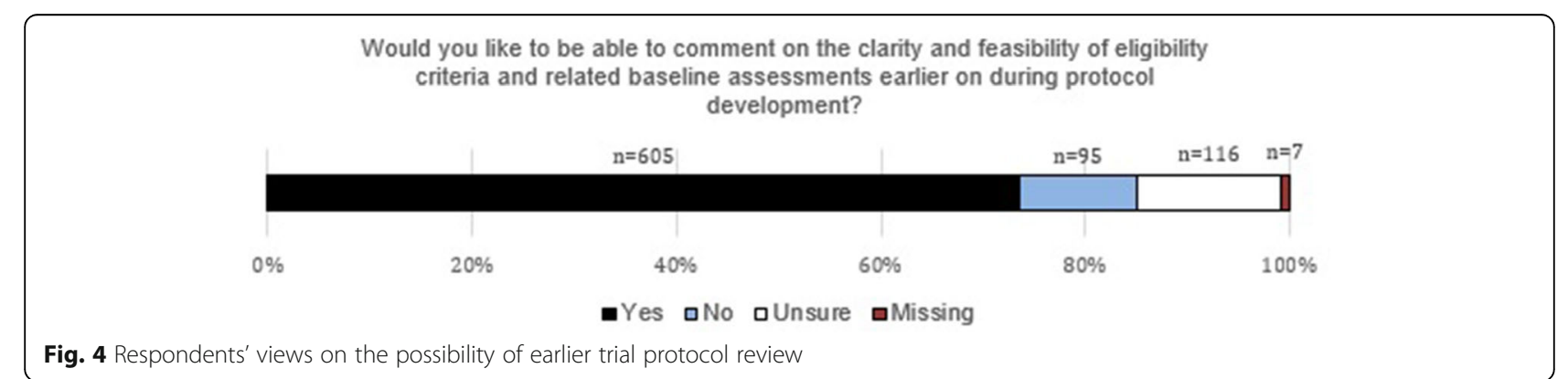

Fig. 4 Respondents' views on the possibility of earlier trial protocol review 
Table 1 Top 10 most frequent responses about types of problems experienced when using eligibility criteria $^{a}$

\begin{tabular}{|c|c|c|c|c|}
\hline Rank & Comment type & $\begin{array}{l}\text { Category (Clarity, Feasibility, } \\
\text { Suitability, Other) }\end{array}$ & $n$ & $\begin{array}{l}\text { Proportion of all } \\
\text { comments }(n=671)\end{array}$ \\
\hline 1 & Criteria generally ambiguous or unclear & Clarity & 297 & $44 \%$ \\
\hline 2 & Tests required in a short timeframe (i.e. difficult to achieve) & Feasibility & 134 & $20 \%$ \\
\hline 3 & Criteria too restrictive & Suitability & 41 & $6 \%$ \\
\hline 4 & Required tests not standardly done locally & Feasibility & 40 & $6 \%$ \\
\hline 5 & $\begin{array}{l}\text { Hard to gather required data in time available (e.g. information hard to locate, } \\
\text { stored in several places) }\end{array}$ & Feasibility & 22 & $3 \%$ \\
\hline 6 & Required timelines unclear & Clarity & 20 & $3 \%$ \\
\hline$=7$ & Difficulty implementing subjective eligibility criteria & Clarity & 19 & $3 \%$ \\
\hline$=7$ & Problems with wording, phrases or terminology used & Clarity & 19 & $3 \%$ \\
\hline 9 & Criteria too complex & Clarity & 18 & $3 \%$ \\
\hline 10 & Unclear which previous treatments allowed & Clarity & 17 & $3 \%$ \\
\hline
\end{tabular}

${ }^{a}$ Excluding comments that could not be categorised as the meaning was not totally clear, $n=33$

Our choice of survey topics was informed by a wider programme of work underway at the Clinical Trials Research Unit (CTRU), University of Leeds, on how to assure the quality of trial eligibility criteria (i.e., to make sure they are well-chosen, wellwritten, and to minimise any risk of classification error [where patients who should be eligible are classified as ineligible or vice versa]). The CTRU is a well-established academic trials unit designing, conducting and analysing trials in a range of areas, such as trials of cancer treatments (including early phase trials [32]), surgical interventions and complex interventions. CTRU runs clinical trials with investigational medicinal products (CTIMPs) and those without (non-CTIMPs). Most CTRU trials are multicentre, mainly recruiting in the UK but with some trials recruiting internationally.
The survey asked respondents about their trial experience in general, not only their experiences working on CTRU trials. It aimed to find out:

- How frequently recruiters encounter problems using eligibility criteria (to inform our understanding of the incidence of problems);

- For those who experience problems, the sorts of problems encountered (free-text description, with categorical questions about whether or not the encountered problems have led to patients being incorrectly included or excluded from trials);

- How eligibility criteria are typically accessed when needed, given that criteria may be available in more than one place (to inform our understanding of how recruiters access information and therefore where best to target any quality improvement efforts);

Table 2 Top 10 most frequent additional comments about how eligibility criteria are developed or used ${ }^{a}$

\begin{tabular}{llll}
\hline Rank & Comment type & n & $\begin{array}{c}\text { Proportion of all meaningful } \\
\text { comments }(\boldsymbol{n}=\mathbf{2 2 9})^{\mathbf{b}}\end{array}$ \\
\hline 1 & Support for earlier review of protocol/eligibility criteria & 66 & $29 \%$ \\
2 & Want clarity/consistency of information & 20 & $9 \%$ \\
3 & Already feedback to sponsor when criteria problematic & 14 & $6 \%$ \\
4 & Criteria are often too restrictive & 12 & $5 \%$ \\
5 & Criteria should be more inclusive of "real-world" patients & 11 & $5 \%$ \\
6 & Support for sponsor-provided eligibility checklists & 9 & $4 \%$ \\
7 & Happy to ask sponsor if have queries about criteria & $3 \%$ \\
$=8$ & Justification for criteria would be useful & 7 & $3 \%$ \\
$=8$ & Criteria can be long and complex & 7 & $3 \%$ \\
$=10$ & Criteria need to be more specific & 6 & $3 \%$ \\
$=10$ & Research Nurse involvement is/could be key in ensuring criteria quality & 6 & $3 \%$ \\
$=10$ & The right people/enough people already tend to be involved in protocol development & 6 & $3 \%$
\end{tabular}

aFull question: "Do you have any other comments about how eligibility criteria are developed or used?"

'Total responses: 282; excluded 51 for containing only "no comment" or similar; excluded 2 for comments on issues unrelated to eligibility criteria in trials 
- The level of interest among recruiters for reviewing eligibility criteria during protocol development, i.e. when there is still a chance to influence the protocol content, as opposed to being presented with a final, approved protocol to implement (in our broader work, this was suggested as a way to improve eligibility criteria and we wanted to gauge interest in this).

The survey also provided space for any other comments about development or use of eligibility criteria.

Potential respondents were eligible for the survey only if they could answer positively to the first survey question: "Are you involved in assessing potential clinical trial participants against protocol eligibility criteria and are you currently working in the UK?"

We collected a limited amount of data about respondents' characteristics (while maintaining individuals' anonymity) to describe them and to enable exploration of any differences between respondent groups. Requested variables were as follows: medical doctor or not, levels of healthcare provided during career, experience working on CTIMPs and/or nonCTIMPs, number of years working on clinical trials and any experience writing trial eligibility criteria. We did not formally test the survey prior to using it, but did ask a recruiter to review it for clarity and appropriateness.

The survey was disseminated by email to professionals collaborating on trials run by the CTRU, with one reminder 2 weeks after the first notification. It was also disseminated through the National Institute for Health Research via relevant mailing lists, Twitter and a newsletter. The message accompanying the survey link (available in the Supplementary Information) said people could share it with other interested individuals. The survey was open between 8th August and 6th September 2019. In line with UK Health Research Authority guidance on proportionate consent [33], consent to participate was presumed to have been given when people chose to complete the survey. The software used to host the survey cannot prevent multiple participation, but we have no strong reason to suspect any individuals would have participated more than once.

Analysis of categorical data was descriptive only, presenting proportions (including for missing responses) with $95 \%$ confidence intervals for the population proportion. We also conducted exploratory subgroup analyses (not defined prior to data collection). As we were looking only to gather some basic information on this topic, we did not define a primary outcome and the survey had no pre-calculated sample size or statistical power (although before the survey we agreed that achieving at least 500 respondents would subjectively constitute success).
Responses to both of the comments fields were summarised via inductive analysis, working without a pre-existing framework to categorise each comment initially at a granular level (i.e. based on its specific contents) then combining these categories into broader themes. We chose this approach as we had neither a prior framework to work with, nor any strong rationale to make prior assumptions about the sorts of comments we would receive. For the comment field about types of problems experienced, coding was double-checked by another author (VN) for a random $10 \%$ of responses. All analysis was conducted by WC in Microsoft Excel.

We did not require ethical approval for this work, according to the Health Research Authority decision tool [34], and we did not collect any personal or confidential data. We have followed the best practice recommendations for reporting this sort of study and include a CROSS checklist with the Supplementary Information [35].

\section{Results}

A total of 823 eligible respondents took part in the survey (total responses: 874). Detailed information on their characteristics can be found in Fig. 1. One third of eligible respondents were not medical doctors, reported only experience in secondary care and had experience both of CTIMPs and non-CTIMPs.

Figure 2 summarises the responses to the quantitative survey questions about frequency of problems experienced when using eligibility criteria. In total, 653 respondents (79\%, 95\% confidence interval $[\mathrm{CI}] 77-82 \%$ ) said that they find problems with eligibility criteria in some trials they work on, and a substantial minority (76 respondents; 9\%, 95\% CI 7-11\%) said they find problems in most trials that they work on. Of the 671 respondents who commented about the most common types of problems, $448(67 \%)$ mentioned issues of clarity (i.e. the meaning of eligibility criteria is not clear), 230 (34\%) mentioned issues of feasibility (i.e. the meaning may be clear, but not achievable in practice, particularly due to required assessments within short timelines) and 91 (14\%) mentioned issues of suitability (i.e. the meaning may be clear and processes may be feasible, but they disagree that criteria are necessary). Some respondents mentioned more than one theme within a single comment. Table 1 shows the top 10 most frequent responses to the free-text question about types of problems experienced.

Exploratory subgroup analyses of the question about frequency of problems showed little difference in responses depending on role (doctor vs non-doctor), or most aspects of experience (secondary care vs no experience in secondary care, experience in writing eligibility criteria vs none, years of trial experience). Respondents 
without CTIMP experience were more likely to say they had never experienced problems (29\% [95\% CI 19-40\%] compared to $9 \%$ [7-11\%] of those with CTIMP experience), a finding that did not seem to be explained by any of the other demographics variables.

Of those who said they find eligibility criteria problems in at least some trials, 197 (27\%, 95\% CI 24$30 \%)$ said these had led to incorrectly included patients and 408 (55\%, 95\% CI 52-59\%) said they had not (18\% unsure, missing or marked "not applicable"). By contrast, 296 (40\%, 95\% CI 37-44\%) of the same respondents said problems had led to incorrectly excluded patients and 252 (34\%, 95\% CI 31-38\%) said they had not (26\% unsure, missing or marked "not applicable").

Responses to the question about accessing eligibility criteria are shown in Fig. 3. A total of 462 respondents (56\%, 95\% CI 53-60\%) said they access eligibility criteria by referring to the trial protocol. A second large group of respondents $(32 \%, 95 \%$ CI $29-36 \%, n=266)$ said their primary method was to use the sponsor-provided eligibility checklists or Case Report Forms (CRFs). Only 9\% (95\% CI $7-11 \%, n=78)$ said they used locally produced forms based on the protocol ("crib sheets").

Responses to the question about earlier review of eligibility criteria are shown in Fig. 4. A substantial majority of respondents (74\%, 95\% CI 70-77\%, $n=605$ ) said they would like to be able to comment on the clarity and feasibility of eligibility criteria and related baseline assessments earlier on in protocol development. Fifteen percent $(n=123)$ were unsure or did not respond to the question, and only $12 \%(n=95)$ said they would not be interested in commenting on protocols earlier in development.

Table 2 shows the top 10 most frequent responses to the "other comments" free-text question. Of the 229 meaningful responses, the commonest themes were further views on problems experienced with eligibility criteria $(69 \%, n=157)$, further support for earlier review of trial protocols $(34 \%, n=78)$ and suggestions that current practice in developing trial protocols is already adequate $(22 \%, n=51)$.

\section{Discussion}

We conducted a simple survey to gather exploratory evidence on clinical trial recruiters' experiences using eligibility criteria. Although we prioritised number of responses over detail, our survey suggests that recruiters often find challenges in implementing eligibility criteria. The comments explaining the sorts of problems that arise suggested that there are three main areas of concern: (1) clarity (unclear exactly what a criterion means), (2) feasibility (clear what a criterion means, but not easily achievable in practice, particularly with regard to required tests and timelines) and (3) appropriateness (clear what criterion means, and criterion and associated tests are achievable, but disagreement or uncertainty about why the criterion is necessary). Previous reports have raised the points on clarity [36] and feasibility [26], including the finding that as many as $7 \%$ of eligibility criteria in clinicaltrials.gov entries were "incomprehensible" [36]. Along with the previous evidence on the occurrence of eligibility classification errors and on unjustifiable exclusions, our results confirm that there is still work to do to improve eligibility criteria in trials. Our results also provide some evidence that there may be more problems in CTIMPs than non-CTIMPs, perhaps arising from the stricter regulatory environment in place in those sorts of trials.

The issue of clarity is perhaps the most likely to lead to classification errors, i.e. eligibility rules inadvertently being interpreted differently to the intentions of the protocol authors, leading to patients either being recruited when they should not have been, or incorrectly excluded on the basis of eligibility. Our survey does not directly provide data on how often errors of this kind occur, though data from other studies suggests they are not uncommon [5, 23-25]. These errors may or may not have a significant effect on trial integrity, but if small changes to how criteria are written could reduce error incidence and subsequently improve trial robustness and efficiency, these seem worthwhile. We should also not ignore the potentially significant impact that errors can have on individuals. Incorrect inclusions can put people's safety at risk, if the breached eligibility criterion is in place to protect wellbeing. Incorrect exclusions can cause people inconvenience or upset, or unfairly deny them the potential benefits of research participation.

The issues of feasibility and appropriateness mentioned in free-text comments perhaps say more about exclusion than inclusion. At least some recruiters feel they are hindered by complex or demanding pretrial procedures, or overly selective criteria. This is also borne out by recruiters more commonly experiencing patients being incorrectly excluded from trials than incorrectly included. This links to the recognised problem of limited generalisability of trial results, observed across various trial settings $[10,19,37-42]$ and for at least the last few decades $[17,43]$. Although other factors may contribute towards limited generalisability (such as recruiter discomfort in approaching some eligible patients [44] or underlying problems with the feasibility of interventions [45]), specific exclusions in eligibility criteria are likely to play a large part.

If the survey respondents' impressions are correct, and incorrect exclusions are truly more common than incorrect inclusions, this may reflect risk aversion on the part of those designing and running trials (i.e. more 
efforts are made to prevent incorrect inclusions). Trialists might well be concerned about incorrect inclusions, as these can have implications for individuals' safety in the short or medium term. However, in the longer term and therefore perhaps less immediately obvious, stricter criteria can have negative effects on trial results' generalisability, trial recruitment [26, 46, 47] (still recognised as a key challenge in running successful trials [48-50]) and access to trial participation. Our work does not provide answers to how to find the correct balance between these competing priorities, and there may be no set of eligibility criteria that satisfies everyone. However, we suggest, as did some survey respondents and as have authors of previous reports [ 1 , $2,51,52]$, that communicating the reasons for choices made in defining eligibility could at least give more transparency for recruiters (and even for potential trial participants [53]). We suggest this is useful for all criteria, even those that seem "self-explanatory" [51], for example in assessing the implications of eligibility classification errors during trial management or analysis (particularly where ineligible patients are entered into a trial). It may also be useful to justify the type of test or assessment for each eligibility criterion, especially where assessments are subjective [54]. If it is inconvenient to have this detail in the main body of the trial protocol, we suggest it could be available in an appendix instead.

The finding that recruiters most often said they referred to eligibility criteria in the protocol, closely followed by eligibility checklist CRFs, contradicted our prior assumption that many might refer to locally prepared "crib sheets" for participant recruitment. This is useful in knowing where best to target efforts for quality assurance and control. It is also helpful because the protocol and CRFs are under trial sponsor control, whereas locally prepared documents might contain errors or inconsistencies (or require additional sponsor or recruiter time in checking that there are no such errors). Others have suggested screening logs might also be a convenient place to list eligibility criteria [55]. Suitable quality control processes are needed to ensure all iterations of the criteria are complete and correct, within the protocol itself and in any other documents [30].

There was strong support among recruiters to have more involvement in reviewing protocols at a time when they could still influence the protocol content. Just as it is becoming ever more common to involve patients in trial design $[56,57]$, it also makes sense to consult, during the design phase, the people who will implement the trial protocol about its contents. This may already happen to some degree, and some survey respondents suggested this. However, it may currently be in a limited way, such as having a research nurse on the trial management group
[58] (although in our experience, this is not always done). The group involved in developing a trial will usually include several clinicians, from separate healthcare centres. There may be an assumption that these clinicians' standard practices and experiences are generalisable beyond their centres. Wider consultation at an earlier stage might helpfully scrutinise this assumption (including on issues like variation in normal lab values across trial centres [59]) and give greater reassurance about the chances of the trial recruiting to target and on time. It may also have benefits in terms of site training [60] or ensuring recruiters are comfortable with applying the eligibility criteria [44].

It is possible that this finding about earlier protocol review is particularly affected by the self-selected nature of the survey sample, i.e. people responding to our survey were more likely to approve of this than those who did not. We cannot discount the influence of selection bias; however, it does show that there is a group of recruiters who would be willing to carry out early reviews (even if this would not answer the further question of whether their views on the eligibility criteria are more broadly representative).

We accept that, in some settings, there may be no recruiters appointed or available during the protocol development stage. This would obviously preclude such an early review. However, in our experience it is reasonably common to have interested trial sites early on in trial setup, either through early engagement with potential recruiters about the new trial, or because the same sites were involved in previous, similar trials with the same sponsor.

Respondents' comments highlighted some other potential challenges to carrying out this early review. For example, there were concerns about the possibility of accommodating different recruiters' views, and scepticism about whether recruiters' views could actually have any influence on protocol design. In particular, a few respondents were concerned about the time additional review would add to trial setup. We suggest a suitable mechanism is conceivable to overcome these barriers. For example, there could be a consultation period whereby a draft protocol is made available to all potential sites for a short period for feedback gathering. An organisation such as the UK Health Research Authority might be in a good position to facilitate such a process, which would be analogous to its pharmacy and radiation assurance schemes [61]. Although this might delay study setup times, it might be justified by reciprocal gains in terms of recruitment success or other efficiencies.

\section{Opportunities for improvement}

Much of the published literature about eligibility criteria covers generalisability issues or "formalisation" of 
criteria for various informatics purposes [62, 63]. The latter group includes methods for using criteria in automated electronic health record data searching, either for finding potentially eligible trial participants $[64,65]$ or assessing trial feasibility [66-68].

There is relatively little prior evidence from a quality perspective, i.e. how to ensure criteria are well-selected, well-written, and not liable to misinterpretation or classification error $[4,5]$. Some of the formalisation work could inform this topic. For example, the requirements for eligibility criteria to be clear and allow only binary responses apply in all settings, regardless of whether criteria are being evaluated by computers or humans. However, some criteria cannot easily be formalised for informatics purposes [69-71] and as long as these purposes are not common practice, we still need methods to assure quality in design and implementation of eligibility criteria in the context of use by human recruiters.

Prior reports about criteria quality suggest expert case review [6, 27], run-in periods [4] and an audit-feedback process [72] might be worthwhile interventions. However, it remains to be seen if any of these methods are scaleable or otherwise generalisable outside the settings they have been tried in, and the evidence supporting their effectiveness could not easily be described as robust.

We suggest all eligibility criteria should contain the same few core elements, namely a clear statement that allows only a "yes" or "no" response, a type of test or assessment (including where this may be subjective, or just checking existing data in medical notes) and a timeline for each trial-specific assessment (e.g. within $x$ days of randomisation). For statements about past medical history, there should also be a timeframe, unless this is unambiguously implied (e.g. use of a gerund such as "breastfeeding" implies this is at the time of assessment). The test and timeline can be elsewhere in the protocol, but placing them beside or even within the eligibility criteria could ensure that these elements are present and that recruiters are fully aware of the requirements. Care should be taken with clarity of all time-related descriptions, particularly regarding exactly which "anchor" in time applies [63, 73, 74]. While publicly available protocol templates give general guidance on writing clear and complete eligibility criteria [75-77], we do not know of any that clearly state the need to include all the elements we have mentioned here. Although they may usually be included in practice, without clear guidance there is a risk they may sometimes be missed, with resulting negative impact on clarity or completeness of criteria.

We have also considered a review process to scrutinise criteria at the draft stage. This would include the review by potential sites, which our survey suggests has strong support from recruiters. It could also include targeted trial and data manager review, and Chief Investigator review to check the criteria are including and excluding the intended groups. Automated methods to compare criteria against those of similar trials may eventually help with this process [67] and this could also be a suitable time to check that the drafted criteria are suitably inclusive [13]. Our experience has been that implementing such a review is challenging, principally because of the lack of an optimal time to conduct it. Protocol development can be complex [78], with iterative drafting continuing until it is ready for its approval submissions, and at that point there can be little appetite to delay further. We therefore recommend building the various aspects of quality into the development process through training, templates and other such mechanisms. Clearly, all our suggestions would need further development and evaluation before being adopted more widely.

\section{Strengths and limitations}

This was a brief, focussed survey with a substantial number of responses that gives weight to its conclusions. Our survey results may not be generalisable beyond UK academic trials, although from free-text comments it was clear that some survey respondents had experience working on commercially sponsored trials. Although survey respondents knew the overall results would be reviewed by the CTRU, individual responses were anonymous so we have no strong reason to suspect the questions were not answered honestly. The results give a clear message about the existence of suboptimal trial eligibility criteria, and willingness among recruiters to be involved in raising standards. The number of responses suggests considerable recruiter interest in this topic. We suggest the emerging themes from our work of clarity, feasibility and suitability may constitute a useful framework for evaluating other clinical trial processes.

We acknowledge several limitations not already mentioned. The survey was exploratory in nature, containing only a few questions because we consciously prioritised obtaining a larger volume of responses over more detail. Further work could collect more detailed data, and/or be statistically powered to answer a more specific research question. Our information on the responders' characteristics could be considered limited, and due to the way the survey was disseminated, we are unable to give a precise survey response rate. Our survey does not provide evidence on the prevalence of classification errors in implementing eligibility criteria, but data on this is available elsewhere (see references already given). We also cannot easily comment on exactly why certain sorts of problems occur, but we 
suggest this could be a good subject for further research in this area.

The invitation message (see Supplementary Information) asked people to contribute even if they did not feel eligibility criteria were problematic. However, we cannot discount the possibility of selection bias in responses. There were no limits on the number of responses per site, so this may have affected the results in ways we cannot easily predict. However, respondents answered as individuals and there seems no strong reason to think that individuals in the same organisation would automatically have the same (or different) views.

Although we got recruiter feedback on the survey during its development, we did not formally validate our survey before use. There is therefore some chance that respondents interpreted the questions in varying ways, or that questions were inadvertently leading (despite our efforts to avoid this).

\section{Conclusions}

The results of our exploratory survey confirm that, in this setting at least, problems for trial recruiters routinely arise from the content and clarity of trial eligibility criteria. These problems can have negative consequences both for trials and for individual patients. Recruiters strongly support the suggestion that they be more involved in protocol development at an earlier stage, although questions remain about exactly how to implement such involvement. Our finding that recruiters rely on sponsor-provided documents for accessing eligibility criteria helps sponsors target their quality assurance activity. We invite other trialists to consider our suggestions for how eligibility criteria should be developed.

\section{Abbreviations}

Cl: Confidence interval; CRF: Case Report Form; CTIMP: Clinical Trial of an Investigational Medicinal Product (non-CTIMP: a trial without an IMP);

CTRU: Clinical Trials Research Unit (University of Leeds)

\section{Supplementary Information}

The online version contains supplementary material available at https://doi. org/10.1186/s13063-021-05723-6.

\section{Additional file 1. \\ Additional file 2. \\ Additional file 3.}

\section{Acknowledgements}

We would like to thank the CTRU trial teams who helped with aspects of the survey and with the wider programme of work, in particular Heather Cook, Ruchi Higham, Samantha Noutch and Heather Poad. We are grateful to Amy Beth Coulson for statistical advice and to Rachael Selby (Sheffield Teaching Hospitals NHS Foundation Trust and University of Sheffield) for providing project feedback from a recruiter's perspective. We also acknowledge the help of Christopher Rhymes, Lead Research Nurse, and Jacqui Dooley, Portfolio Delivery Facilitator, both of the NIHR Clinical Research Network Yorkshire and Humber, for their help in disseminating the survey. We would also like to thank all those who gave a moment of their time to contribute to the survey and anyone else who supported this work in various other ways.

\section{Authors' contributions}

WJC initiated the work, drafted and coordinated the survey, analysed the data, produced the initial draft of the paper and coordinated development of all subsequent drafts. The other authors helped design the survey, interpreted the survey results, reviewed manuscript drafts and agreed the final manuscript text. VN carried out the double-check of the analysis of the free-text comments data. WJC, KM, JBO, RS, CT and VN participated in an internal trials unit working group about eligibility criteria development, the outcomes of which have informed the design and interpretation of this work. All authors read and approved the final manuscript.

\section{Funding}

This work was supported by a Cancer Research UK Core CTU infrastructure grant (reference A24929). The funder had no role in the survey design, implementation or analysis.

\section{Availability of data and materials}

The survey data supporting this work are available on reasonable request. Requests should be directed to the corresponding author in the first instance (w.cragg@leeds.ac.uk).

\section{Declarations}

Ethics approval and consent to participate Not applicable.

Consent for publication

Not applicable.

\section{Competing interests}

The authors declare that they have no competing interests.

Received: 10 August 2021 Accepted: 13 October 2021

Published online: 24 October 2021

\section{References}

1. Chan A-W, Tetzlaff JM, Gøtzsche PC, et al. SPIRIT 2013 explanation and elaboration: guidance for protocols of clinical trials. BMJ. 2013;346(9):e7586. https://doi.org/10.1136/bmj.e7586.

2. Kim ES, Bruinooge SS, Roberts S, Ison G, Lin NU, Gore L, et al. Broadening eligibility criteria to make clinical trials more representative: American Society of Clinical Oncology and Friends of Cancer Research Joint Research statement. J Clin Oncol. 2017;35(33):3737-44. https://doi.org/10.1200/JCO.2 017.73 .7916

3. Treweek S, Zwarenstein M. Making trials matter: pragmatic and explanatory trials and the problem of applicability. Trials. 2009;10(1):37. https://doi.org/1 0.1186/1745-6215-10-37.

4. Simpson F, Sweetman EA, Doig GS. A systematic review of techniques and interventions for improving adherence to inclusion and exclusion criteria during enrolment into randomised controlled trials. Trials. 2010;11(1). https://doi.org/10.1186/1745-6215-11-17.

5. Yelland LN, Kahan BC, Dent E, Lee KJ, Voysey M, Forbes AB, et al. Prevalence and reporting of recruitment, randomisation and treatment errors in clinical trials: a systematic review. Clin Trials. 2018;(3):278-85. https://doi.org/10.11 77/1740774518761627.

6. Vining RD, Salsbury SA, Pohlman KA. Eligibility determination for clinical trials: development of a case review process at a chiropractic research center. Trials. 2014;15(1). https://doi.org/10.1186/1745-6215-15-406.

7. Rehman AM, Ferrand R, Allen E, Simms V, McHugh G, Weiss HA. Exclusion of enrolled participants in randomised controlled trials: what to do with ineligible participants? BMJ Open. 2020;10(12):e039546. https://doi.org/1 0.1136/bmjopen-2020-039546.

8. Yelland LN, Sullivan TR, Voysey M, Lee K, Cook JA, Forbes AB. Applying the intention-to-treat principle in practice: guidance on handling randomisation errors. Clin Trials. 2015;12(4):418-23. https://doi.org/10.1177/174077451 5588097. 
9. Lösch C, Neuhäuser M. The statistical analysis of a clinical trial when a protocol amendment changed the inclusion criteria. BMC Med Res Methodol. 2008;8(1). https://doi.org/10.1186/1471-2288-8-16.

10. He J, Morales DR, Guthrie B. Exclusion rates in randomized controlled trials of treatments for physical conditions: a systematic review. Trials. 2020;21(1): 228. https://doi.org/10.1186/s13063-020-4139-0.

11. King NMP. Defining and describing benefit appropriately in clinical trials. J Law, Med Ethics. 2000;28(4):332-43. https://doi.org/10.1111/j.1748-720X.2 000.tb00685.x

12. Benefits of taking part in research - Participating in research Understanding dementia research - Research - Alzheimer Europe. 2013. https://www.alzheimer-europe.org/Research/Understanding-dementia-resea rch/Participating-in-research/Benefits-of-taking-part-in-research. Accessed 15 July 2020.

13. Witham MD, Anderson E, Carroll CB, Dark PM, Down K, Hall AS, et al. Ensuring that COVID-19 research is inclusive: guidance from the NIHR INCLUDE project. BMJ Open. 2020;10(11):e043634. https://doi.org/10.1136/ bmjopen-2020-043634.

14. Hilgers RD, Manolov M, Heussen N, Rosenberger WF. Design and analysis of stratified clinical trials in the presence of bias. Stat Methods Med Res. 2020; 29(6):1715-27. https://doi.org/10.1177/0962280219846146.

15. George SL. Reducing patient eligibility criteria in cancer clinical trials. J Clin Oncol. 1996;14(4):1364-70. http://www.ncbi.nlm.nih.gov/pubmed/8648395. . https://doi.org/10.1200/JCO.1996.14.4.1364

16. Buyse ME. The case of loose inclusion criteria in clinical trials. Acta Chir Belg. 90(3):129-31 http://www.ncbi.nlm.nih.gov/pubmed/2375213.

17. Yusuf S, Held P, Teo KK, Toretsky ER. Selection of patients for randomized controlled trials: implications of wide or narrow eligibility criteria. Stat Med. 1990;9(1-2):73-86. https://doi.org/10.1002/sim.4780090114.

18. Zhang S, Liang F, Li W, Tannock I. Comparison of eligibility criteria between protocols, registries, and publications of cancer clinical trials. J Natl Cancer Inst. 2016;108(11):djw129. https://doi.org/10.1093/jnci/djw129.

19. Gandhi M, Ameli N, Bacchetti P, Sharp GB, French AL, Young M, et al. Eligibility criteria for HIV clinical trials and generalizability of results: the gap between published reports and study protocols. AIDS. 2005;19(16):1885-96. https://doi.org/10.1097/01.aids.0000189866.67182.f7.

20. Blümle A, Meerpohl JJ, Rücker G, Antes G, Schumacher M, Von Elm E. Reporting of eligibility criteria of randomised trials: cohort study comparing trial protocols with subsequent articles. BMJ. 2011;342(7805). https://doi. org/10.1136/bmj.d1828.

21. Getz KA, Zuckerman R, Cropp AB, Hindle AL, Krauss R, Kaitin Kl. Measuring the incidence, causes, and repercussions of protocol amendments. Drug Inf J. 2011;45(3):265-75. https://doi.org/10.1177/009286151104500307.

22. Willoughby C, Fridsma D, Chatterjee L, Speakman J, Evans J, Kush R. A Standard Computable Clinical Trial Protocol: the role of the BRIDG Model. Drug Inf J. 2007;41(3):383-92. https://doi.org/10.1177/009286150704100312.

23. Stenning SP, Cragg WJ, Joffe N, Diaz-Montana C, Choudhury R, Sydes MR, et al. Triggered or routine site monitoring visits for randomised controlled trials: results of TEMPER, a prospective, matched-pair study. Clin Trials. 2018. https://doi.org/10.1177/1740774518793379.

24. Brosteanu O, Schwarz G, Houben P, Paulus U, Strenge-Hesse A, Zettelmeyer $U$, et al. Risk-adapted monitoring is not inferior to extensive on-site monitoring: results of the ADAMON cluster-randomised study. Clin Trials. 2017;14(6):584-96. https://doi.org/10.1177/1740774517724165.

25. Wyman Engen N, Huppler Hullsiek K, Belloso WH, Finley E, Hudson F,

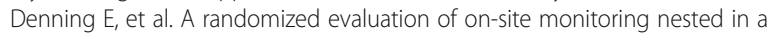
multinational randomized trial. Clin Trials. 2019;(1):3-14. https://doi.org/10.11 77/1740774519881616.

26. Statler A, Othus M, Erba HP, Chauncey TR, Radich JP, Coutre S, et al. Comparable outcomes of patients eligible vs ineligible for SWOG leukemia studies. Blood. 2018;131(25):2782-8. https://doi.org/10.1182/blood-2018-01826693.

27. Spragg RG, Masys DR, Sergeant D, Lawrie T, Taut FJH. An informatics strategy to assure enrollment criteria compliance in studies of the critically ill. Contemp Clin Trials. 2010;31(6):530-5. https://doi.org/10.1016/j.cct.2010. 07.006.

28. Sweetman EA, Doig GS. Failure to report protocol violations in clinical trials: a threat to internal validity? Trials. 2011;12(1):214. https://doi.org/10.1186/1 745-6215-12-214.

29. Meeker-O'Connell A, Glessner C, Behm M, Mulinde J, Roach N, Sweeney F, et al. Enhancing clinical evidence by proactively building quality into clinical trials. Clin Trials. 2016;13(4):439-44. http://ctj.sagepub.com/content/13/4/43 9.abstract. https://doi.org/10.1177/1740774516643491.

30. MHRA. Good Clinical Practice Guide. Printed in the United Kingdom for The Stationary Office; 2012.

31. Jisc. Online surveys. https://www.onlinesurveys.ac.uk/. Accessed 10 Aug 2021.

32. Brown SR, Sherratt D, Booth G, Brown J, Collinson F, Gregory W, et al. Experiences of establishing an academic early phase clinical trials unit. Clin Trials. 2017;(4):349-56. https://doi.org/10.1177/1740774517710250.

33. Health Research Authority. Informing participants and seeking consent Health Research Authority. 2019. https://www.hra.nhs.uk/planning-andimproving-research/best-practice/informing-participants-and-seekingconsent/. Accessed 3 March 2021.

34. Health Research Authority. Do I need NHS Ethics approval?. 2020. http:// www.hra-decisiontools.org.uk/ethics/index.html. Accessed 3 July 2018.

35. Sharma A, Minh Duc NT, Luu Lam Thang T, Nam NH, Ng SJ, Abbas KS, et al. A Consensus-Based Checklist for Reporting of Survey Studies (CROSS). J Gen Intern Med 2021. 2021;36(10):1-9. https://doi.org/10.1 007/S11606-021-06737-1.

36. Ross J, Tu S, Carini S, Sim I. Analysis of eligibility criteria complexity in clinical trials. Summit on Translat Bioinforma. 2010;2010:46-50 http://www. ncbi.nlm.nih.gov/pubmed/21347148.

37. Jin S, Pazdur R, Sridhara R. Re-evaluating eligibility criteria for oncology clinical trials: analysis of investigational new drug applications in 2015. J Clin Oncol. 2017:35(33):3745-52. https://doi.org/10.1200/JCO.2017.73.4186.

38. Ivie RMJ, Vail EA, Wunsch H, Goldklang MP, Fowler R, Moitra VK. Patient eligibility for randomized controlled trials in critical care medicine: an international two-center observational study. Crit Care Med. 2017;45(2):21624. https://doi.org/10.1097/CCM.0000000000002061.

39. Chen X, Schaufelberger M, Fu M. The eligible population of the PARADIGM$\mathrm{HF}$ trial in a real-world outpatient clinic and its cardiovascular risk between 2005 and 2016. J Cardiovasc Med. 2020;21(1):6-12. https://doi.org/10.2459/ JCM.0000000000000889.

40. Ayaz-Shah AA, Hussain S, Knight SR. Do clinical trials reflect reality? A systematic review of inclusion/exclusion criteria in trials of renal transplant immunosuppression. Transpl Int. 2018;31(4):353-60. https://doi.org/10.1111/ tri.13109.

41. Zimmerman M, Balling C, Chelminski I, Dalrymple K. Have treatment studies of depression become even less generalizable applying the inclusion and exclusion criteria in placebo-controlled antidepressant efficacy trials published over 20 years to a clinical sample. Psychother Psychosom. 2019; 88(3):165-70. https://doi.org/10.1159/000499917.

42. Du Vaure CB, Dechartres A, Battin C, Ravaud P, Boutron I. Exclusion of patients with concomitant chronic conditions in ongoing randomised controlled trials targeting 10 common chronic conditions and registered at ClinicalTrials.gov: a systematic review of registration details. BMJ Open. 2016; 6(9). https://doi.org/10.1136/bmjopen-2016-012265.

43. Begg CB, Engstrom PF. Eligibility and extrapolation in cancer clinical trials. J Clin Oncol. 1987;5(6):962-8. https://doi.org/10.1200/JC0.1987.5.6.962.

44. Donovan JL, Paramasivan S, de Salis I, Toerien M. Clear obstacles and hidden challenges: understanding recruiter perspectives in six pragmatic randomised controlled trials. Trials. 2014;15(1):5. https://doi.org/10.1186/174 5-6215-15-5.

45. Ugalde A, Kiss N, Livingston PM, Rankin N. Commentary on 'Exclusion rates in randomized trials of treatments for physical conditions: a systematic review.'. Trials. 2021;22(1):76. https://doi.org/10.1186/s13063021-05019-9.

46. Printz $C$. The number of patients with lung cancer eligible for a clinical trial could double with broader enrollment criteria. Cancer. 2019;125(22):3907. https://doi.org/10.1002/cncr.32589.

47. Briel M, Speich B, von Elm E, Gloy V. Comparison of randomized controlled trials discontinued or revised for poor recruitment and completed trials with the same research question: a matched qualitative study. Trials. 2019; 20(1):800. https://doi.org/10.1186/s13063-019-3957-4.

48. Duley L, Gillman A, Duggan M, Belson S, Knox J, McDonald A, et al. What are the main inefficiencies in trial conduct: a survey of UKCRC registered clinical trials units in the UK. Trials. 2018;19(1):15. https://doi.org/10.1186/s13 063-017-2378-5.

49. Carlisle B, Kimmelman J, Ramsay T, MacKinnon N. Unsuccessful trial accrual and human subjects protections: an empirical analysis of recently closed trials. Clin Trials. 2015;12(1):77-83. https://doi.org/10.1177/1740774514558307. 
50. Walters SJ, Bonacho dos Anjos Henriques-Cadby I, Bortolami O, et al. Recruitment and retention of participants in randomised controlled trials: a review of trials funded and published by the United Kingdom Health Technology Assessment Programme. BMJ Open. 2017;7(3):e015276. https:// doi.org/10.1136/bmjopen-2016-015276.

51. Schmidt AF, Groenwold RHH, Van Delden JJM, et al. Justification of exclusion criteria was underreported in a review of cardiovascular trials. J Clin Epidemiol. 2014;67(6):635-44. https://doi.org/10.1016/j.jclinepi.2013.12.005.

52. Van Spall HGC, Toren A, Kiss A, Fowler RA. Eligibility criteria of randomized controlled trials published in high-impact general medical journals: a systematic sampling review. J Am Med Assoc. 2007;297(11):1233-40. https:// doi.org/10.1001/jama.297.11.1233.

53. US Food and Drug Administration. Public Workshop: Evaluating Inclusion and Exclusion Criteria in Clinical Trials IN CLINICAL TRIALS.; 2018. https:// www.fda.gov/media/134754/download.

54. Weijer C, Freedman B, Shapiro S, Fuks A, Skrutkowska M, Sigurjonsdottir M. Assessing the interpretation of criteria for clinical trial eligibility: a survey of oncology investigators. Clin Invest Med. 1998;21(1):17-26.

55. Wilson C, Rooshenas L, Paramasivan S, Elliott D, Jepson M, Strong S, et al. Development of a framework to improve the process of recruitment to randomised controlled trials (RCTs): the SEAR (Screened, Eligible, Approached, Randomised) framework. Trials. 2018;19(1):50. https://doi.org/ 0.1186/s13063-017-2413-6.

56. Crocker JC, Ricci-Cabello I, Parker A, Hirst JA, Chant A, Petit-Zeman S, et al. Impact of patient and public involvement on enrolment and retention in clinical trials: systematic review and meta-analysis. BMJ. 2018;363:k4738. https://doi.org/10.1136/BMJ.K4738.

57. Price A, Albarqouni L, Kirkpatrick J, Clarke M, Liew SM, Roberts N, et al. Patient and public involvement in the design of clinical trials: an overview of systematic reviews. J Eval Clin Pract. 2018;24(1):240-53. https://doi.org/1 $0.1111 /$ jep.12805.

58. Clinical Trials Toolkit - Glossary. https://www.ct-toolkit.ac.uk/glossary/?letter $=$ T\&postcategory = -1. Accessed 24 Feb 2021.

59. Vax Report - Researchers establish new enrollment criteria for African volunteers. https://www.vaxreport.org/vax-5-8-august-2007/677-resea rchers-establish-new-enrollment-criteria-for-african-volunteers. Accessed 1 March 2021.

60. Bechtel J, Chuck T, Forrest A, Hildebrand C, Panhuis J, Pattee SR, et al. Improving the quality conduct and efficiency of clinical trials with training recommendations for preparedness and qualification of investigators and delegates. Contemp Clin Trials. 2020;89:105918. https://doi.org/10.1016/j. cct.2019.105918.

61. What approvals and decisions do I need? - Health Research Authority https://www.hra.nhs.uk/approvals-amendments/what-approvals-do-i-need/. Accessed February 24, 2021.

62. Bhattacharya S, Cantor MN. Analysis of eligibility criteria representation in industry-standard clinical trial protocols. J Biomed Inform. 2013;46(5):805-13. https://doi.org/10.1016/j.jbi.2013.06.001.

63. Chondrogiannis E, Andronikou V, Tagaris A, Karanastasis E, Varvarigou T, Tsuji M. A novel semantic representation for eligibility criteria in clinical trials. J Biomed Inform. 2017;69:10-23. https://doi.org/10.1016/j.jbi.2017. 03.013.

64. Weng C, Tu SW, Sim I, Richesson R. Formal representation of eligibility criteria: A literature review. J Biomed Inform. 2010;43(3):451-67. https://doi. org/10.1016/j.jbi.2009.12.004

65. Gulden C, Landerer I, Nassirian A, Altun FB, Andrae J. Extraction and prevalence of structured data elements in free-text clinical trial eligibility criteria. Stud Heal Technol Inf. 2019:258:226-30.

66. Sumi E, Teramukai S, Yamamoto K, Satoh M, Yamanaka K, Yokode M. The correlation between the number of eligible patients in routine clinical practice and the low recruitment level in clinical trials: a retrospective study using electronic medical records. Trials. 2013;14(1):426. https://doi.org/10.11 86/1745-6215-14-426.

67. Milian K, Hoekstra R, Bucur A, ten Teije A, van Harmelen F, Paulissen J. Enhancing reuse of structured eligibility criteria and supporting their relaxation. J Biomed Inform. 2015;56:205-19. https://doi.org/10.1016/j.jbi.201 5.05.005.

68. Minnerup J, Trinczek B, Storck M, Hohenberger M, Wilpsbäumer S, AbdulRahim AH, et al. Feasibility platform for stroke studies: an online tool to improve eligibility criteria for clinical trials. Stroke. 2015;46(1):137-42. https:// doi.org/10.1161/STROKEAHA.114.007124.
69. Wang AY, Lancaster WJ, Wyatt MC, Rasmussen LV, Fort DG, Cimino JJ. Classifying clinical trial eligibility criteria to facilitate phased cohort identification using clinical data repositories. AMIA Annu Symp Proc. 2018, 2017:1754-63.

70. Köpcke F, Trinczek B, Majeed RW, et al. Evaluation of data completeness in the electronic health record for the purpose of patient recruitment into clinical trials: a retrospective analysis of element presence. BMC Med Inform Decis Mak. 2013;13(1). https://doi.org/10.1186/1472-6947-13-37.

71. Melzer G, Maiwald T, Prokosch HU, Ganslandt T. Leveraging real-world data for the selection of relevant eligibility criteria for the implementation of electronic recruitment support in clinical trials. Appl Clin Inform. 2021;12(1): 17-26. https://doi.org/10.1055/s-0040-1721010.

72. Roos DE, Davis SR, O'Brien PC, Hoskin PJ, Spry NA, Burmeister BH, et al. Eligibility audits for the randomized neuropathic bone pain trial (TROG 96. 05). Australas Radiol. 2000;44(3):303-7. https://doi.org/10.1046/j.1440-1673.2 000.00818.x

73. Boland MR, Tu SW, Carini S, Sim I, Weng C. EliXR-TIME: a temporal knowledge representation for clinical research eligibility criteria. AMIA Jt Summits Transl Sci Proc. 2012;2012:71-80 http://www.ncbi.nlm.nih.gov/ pubmed/22779055.

74. Luo Z, Johnson SB, Lai AM, Weng C. Extracting temporal constraints from clinical research eligibility criteria using conditional random fields. AMIA Annu Symp Proc. 2011:843-52.

75. Health Research Authority. Protocol - Health Research Authority. 2018 https://www.hra.nhs.uk/planning-and-improving-research/research-pla nning/protocol/. Accessed 2 March 2021.

76. National Institutes of Health. Protocol Templates for Clinical Trials |. 2021. grants.nih.gov. https://grants.nih.gov/policy/clinical-trials/protocol-template. htm. Accessed 2 March 2021

77. SPIRIT Group. SPIRIT Electronic Protocol Tool \& Resource (SEPTRE). https:// www.spirit-statement.org/trial-protocol-template/.

78. Gennari JH, Weng C, MCDonald DW, Benedetti J, Green S. An ethnographic study of collaborative clinical trial protocol writing. Stud Heal Technol Inf. 2004;107(Pt2):1461-5.

\section{Publisher's Note}

Springer Nature remains neutral with regard to jurisdictional claims in published maps and institutional affiliations.

\section{Ready to submit your research? Choose BMC and benefit from:}

- fast, convenient online submission

- thorough peer review by experienced researchers in your field

- rapid publication on acceptance

- support for research data, including large and complex data types

- gold Open Access which fosters wider collaboration and increased citations

- maximum visibility for your research: over $100 \mathrm{M}$ website views per year

At $\mathrm{BMC}$, research is always in progress.

Learn more biomedcentral.com/submission 